giornate/paziente $(\mathrm{gg} / \mathrm{pz})$ degli ospedali considerati (fonte S.I.O., A.S.P. Lazio). I tassi sono serviti per stimare gli isolamenti annuali di Gram (-) nella città. È stato valutato l'impatto economico di tali episodi infettivi, moltiplicando per livelli crescenti di spesa $(5000,10000,15000,20000 €)$, ottenuti da indicazioni bibliografiche europee e statunitensi.

Risultati. Gli isolamenti ematici di batteri Gram (-) sono variati da 253 (2001) a 410 (2003), con una media annuale di 319 +/-5.13 (i.c.: 95\%). Gli ospedali valutati hanno totalizzato, nel periodo, oltre $3 * 10^{6} \mathrm{di} \mathrm{gg} / \mathrm{pz}(\sim 26 \%$ delle degenze ordinarie dell'area di Roma). I tassi di incidenza variano da $0.28 / 1000 \mathrm{gg} / \mathrm{pz}$ (2001) a 0.38/1000 gg/pz (2003), con una media triennale di $0.30 / 1000 \mathrm{gg} / \mathrm{pz}$.; si osserva un trend di aumento dei casi. I tassi corrispondono a una media di 1231 batteriemie/sepsi da Gram (-) nella città, per anno (valore medio calcolato sul triennio; da 1211 a 1251 episodi; i.c. $95 \%$ ). La stima del costo oscilla da $\sim 6$ a $\sim 25$ milioni $€$ (valori non aggiustati per la mortalità).

Conclusioni. Dati di letteratura indicano che $\sim 35 \%$ delle sepsi con emocoltura positiva sono sostenute da Gram (-). I tassi presentati sono un esperimento, basato su dati obiettivi, di valutare la rilevanza di tali infezioni nell'area di Roma. Pur con alcuni limiti - in particolare quello di omettere gli episodi per i quali non è stata effettuata una coltura - la stima fornisce risultati utili per la programmazione di interventi sanitari.

\title{
155
}

\section{BATTERIEMIE E SEPSI DA GRAM NEGATIVI NEGLI OSPEDALI DI ROMA: VALUTAZIONE D'IMPATTO EPIDEMIOLOGICO ED ECONOMICO.}

Meledandri M.', Ballardini M.', Falco S. ${ }^{2}$, Carletti M. ${ }^{3}$, Bernaschi P. ${ }^{3}$, Raponi G. ${ }^{4}$, Gallo M.T. ${ }^{5}$, Fontana C. ${ }^{6}$, Testore G.P. ${ }^{6}$ (Fondazione SEERBIO)

\section{'ACO S.Filippo Neri, ${ }^{2} \mathrm{ASL}$ RMC, ${ }^{3}$ RCCS Bambino Gesù, ${ }^{4} \mathrm{AU}$ Policlinico Umberto I, ${ }^{5} \mathrm{RCCS}$ IFO, ${ }^{6} \mathrm{AU}$ Policlinico Tor Vergata.}

Introduzione. Batteriemie e sepsi da batteri Gram (-) sono associate ad un'elevata mortalità cruda e ad alti costi assistenziali. La valutazione del loro impatto sul sistema sanitario è resa difficile dall'assenza - su base nazionale - di una sorveglianza continua delle infezioni ospedaliere. Nel presente lavoro si è posto l'obiettivo di stimare l'incidenza e il costo di tali infezioni nella città di Roma, usando il database della Fondazione SEERBIO. Tale banca dati raccoglie - in forma anonima - gli isolamenti microbici effettuati in diversi ospedali e policlinici universitari della città.

Metodi. Analisi retrospettiva delle emocolture positive di 7 ospedali di Roma nel periodo 2001-2003. Sono stati considerati gli isolamenti di batteri Gram (-) solo se associati ad antibiogramma. Sono stati calcolati i tassi di incidenza annuali e quello medio triennale, rispetto alle 